Radiologe $2019 \cdot 59: 234-241$

https://doi.org/10.1007/s00117-018-0471-8

Online publiziert: 19. November 2018

(c) Der/die Autor(en) 2018

CrossMark

C. Czerny ${ }^{1}$ - C. Chiari ${ }^{2}$ I. Nöbauer-Huhmann ${ }^{1}$

${ }^{1}$ Abteilung für Neuroradiologie/Muskuloskelettale Radiologie, Universitätsklinik für Radiologie und Nuklearmedizin, AKH Wien, Wien, Österreich

${ }^{2}$ Universitätsklinik für Orthopädie, AKH Wien, Wien, Österreich

\title{
Labrumpathologie des Hüftgelenkes
}

- führt damit zu einer gleichmäßigeren Druckverteilung im Hüftgelenk,

- kann Krafteinwirkungen absorbieren und

- erhöht die Stabilität des Gelenkes [2]. zugenommen. Am besten sind die Pathologien des Labrum acetabulare mit der MR-Arthrographie (MRATG) zu erfassen. Neben Labrumveränderungen spielen aber auch Knorpelschädigungen, die auch mit Labrumläsionen - am chondrolabralen Übergang - einhergehen können, eine zunehmende Rolle in der Diagnostik. Mit der MR-ATG sind Einrisse und Abrisse des Labrums durch die Distension der intraartikulären Strukturen gut erkennbar. Jüngst wurde - zur noch besseren Darstellung des chondrolabralen Überganges und von Läsionen in diesem Bereich - die MR-ATG unter gleichzeitiger Traktion der zu untersuchenden Extremität eingesetzt. Veränderungen in diesem Bereich wurden durch die ausgeprägtere Distension des Hüftgelenkes und seiner intraartikulären Strukturen unter Umständen noch besser erkennbar als mit der MR-ATG ohne Traktion. Dies führte auch zu einer neuen modifizierten Einteilung der Labrumläsionen des Hüftgelenkes.

\section{Hintergrund}

Das Labrum des Hüftgelenkes erfüllt mehrere wichtige Funktionen: Es

- erhöht die Kontaktfläche und die Kongruenz der Gleitlager,

- trägt zur Verteilung der Gelenkflüssigkeit bei,

Diese Effekte werden vor allem durch das Vakuumsegel und den Sog zwischen Femurkopf und Azetabulum erzielt, wofür ein intaktes Labrum Voraussetzung ist.

Hüftschmerzen als Symptom können viele Ursachen haben. Die bildgebende Abklärung erfolgt mit Projektionsradiographie, Computertomographie (CT) und MRT. Läsionen des Labrum acetabulare oder freie Gelenkskörper können ebenfalls Hüftschmerzen und ein sog. "giving way" - bei Belastung der betroffenen Extremität wird diese aufgrund des Schmerzes entlastet - verursachen. Die radiologische Abklärung dieser Entitäten erfolgt (in Zusammenschau mit dem Projektionsröntgen) am besten mit der MRT und hier mit der MR-Arthrographie $[3,17,20]$.

Labrumveränderungen können durch degenerative Hüftgelenkserkrankungen
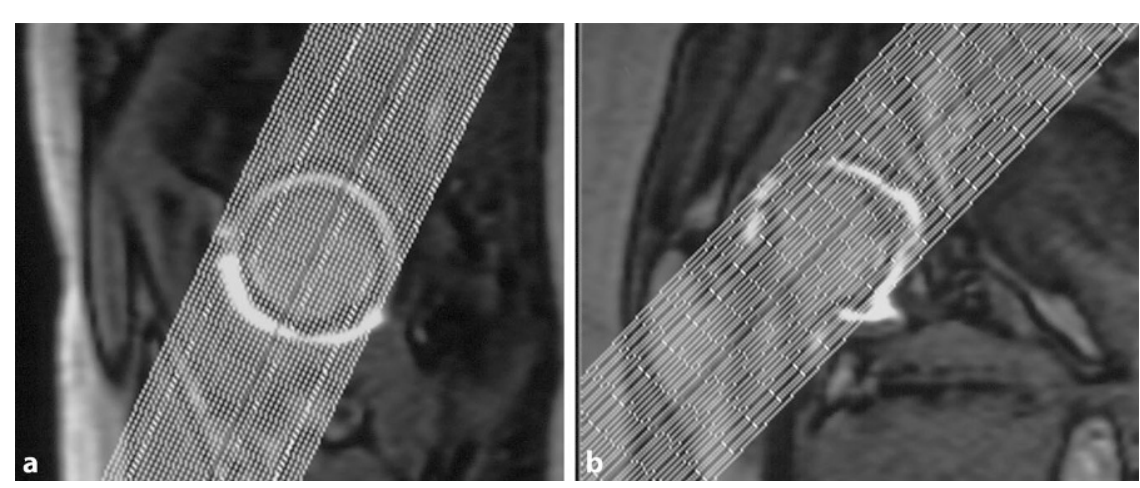

Abb. $1 \Delta$ a Die Abbildung zeigt die Positionierung der schräg-koronalen Schichten. b Die Abbildung zeigt die Positionierung der schräg-sagittalen Schichten

bedingt sein bzw. die Degeneration kann diese noch weiter verschlechtern [9, 21]. Labrumläsionen können aber auch durch ein akutes Trauma oder durch repetitive Mikrotraumata verursacht sein, z. B. bei Patienten unter dem klinischen Bild eines Impingements [14]. Auch Hüftdysplasien oder verminderte Kapselspannung sind prädisponierende Faktoren.

Die Hauptindikation zur MR-Arthrographie des Hüftgelenkes ist die klinisch vermutete Läsion des Labrum acetabulare $[3,4]$. Es kann jedoch selbst bei genauer klinisch-orthopädischen Untersuchung schwierig sein, eindeutig eine Labrumläsion von anderen Krankheitsbildern zu unterscheiden. Eine MR-Arthrographie sollte allerdings nur nach einer genauen fachärztlichen Untersuchung mit gezielter Fragestellung durchgeführt werden $[3-5,8,12]$.

Weitere Indikationen zur MR-Arthrographie sind unter anderem der Verdacht auf Knorpelschädigungen oder freie Gelenkskörper, also Entitäten, die sich mit anderen bildgebenden Methoden nur 

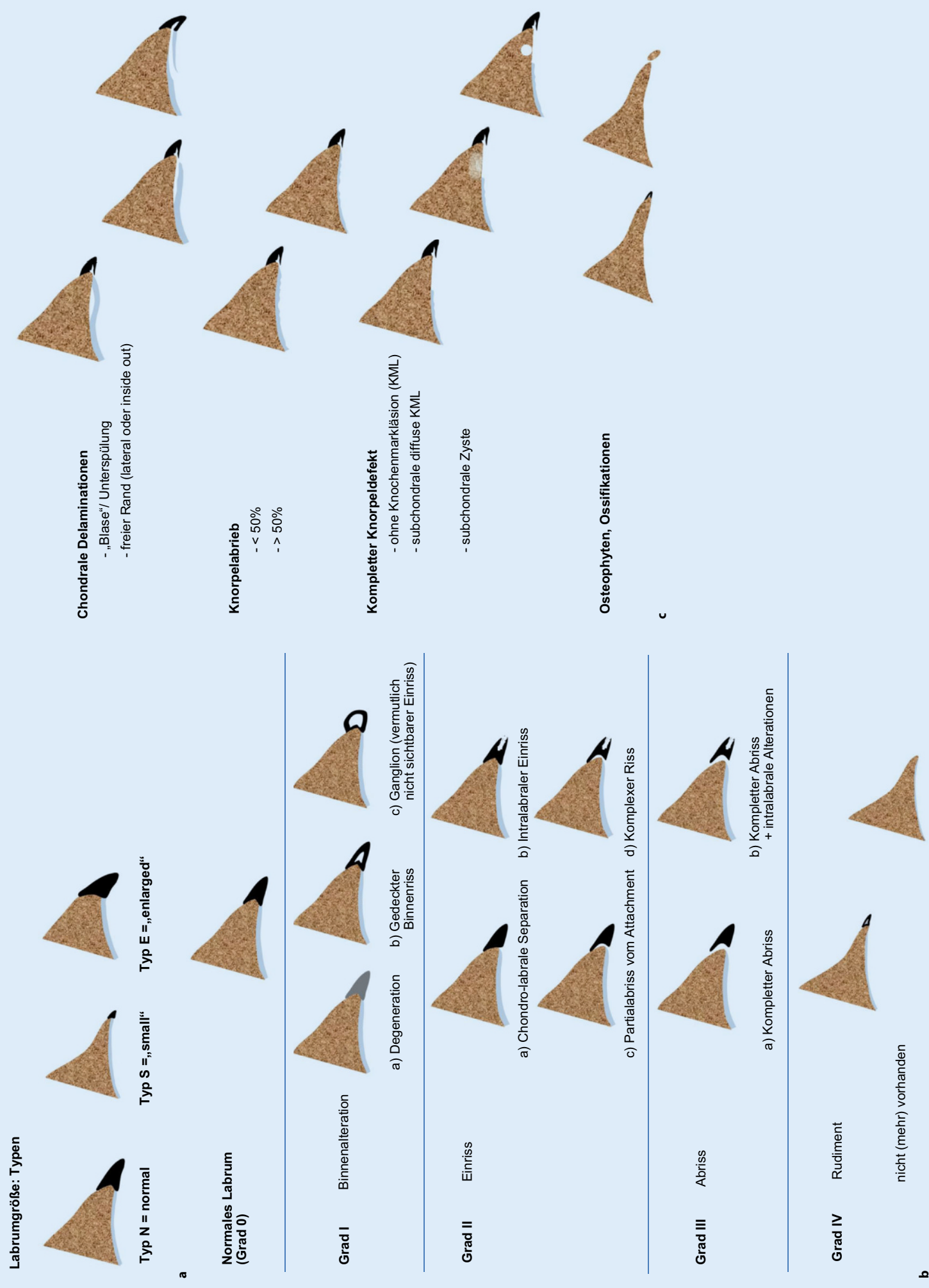

Abb. $2 \Delta$ a Labrumtypen nach der neuen Klassifikation von Czerny/Nöbauer. b Klassifikation der Labrumläsionen nach der neuen Graduierung nach Czerny/Nöbauer nach der modifizierten Einteilung von Schmaranzer auf Basis der MAHORN-Klassifikation. c Einteilung der Veränderungen des labrumnahen Knorpels und Knochens nach der neuen Klassifikation von Czerny/ Nöbauer auf Basis der modifizierten Klassifikation nach Schmaranzer. (Abgebildet ist zur Vereinfachung beispielhaft eine Labrumrissform; diese ist unabhängig wie in $\mathbf{b}$ angegeben zu beschreiben) 
schwer oder nicht nachweisen lassen $[10,11,15]$. Die detaillierte Darstellung und Einschätzung des Knorpelschadens hat besondere Bedeutung für die Indikationsstellung hüftgelenkserhaltender Eingriffe wie Beckenosteotomien zur Behandlung von Hüftdysplasien und deren Abgrenzung zur Implantation einer Totalendoprothese. Die Erfolgschancen stehen in direktem Zusammenhang mit dem präoperativen Zustand des Knorpels. Aufgrund der Übersichtlichkeit dieser Arbeit, welche sich nur mit der Pathologie des Labrum acetabulare beschäftigt, kann auf die Knorpelveränderungen nur vereinzelt und nicht detailliert eingegangen werden.

\section{Untersuchungstechnik}

Die MRT - vor allem die MRT unter Verwendung dedizierter Spulen und mit 3T-MRT - erlaubt unter Umständen eine sehr gute Diagnostik labraler Läsionen. Die MR-Arthrographie - durch die intraartikuläre Kontrastmittel(KM)-Gabe - führt zu einer Distension des Gelenkes und zu einer besseren Erkennbarkeit labraler und knorpeliger Veränderungen [13]. In den letzten Jahren wurde zusätzlich noch die MR-Arthrographie unter Verwendung einer Traktion der zu untersuchenden Extremität durchgeführt. Hierzu wurde eine eigene MR-taugliche Vorrichtung entwickelt, die durch die Variation von Gewichten eine Traktion ermöglicht [20]. Hierdurch kommt es zu einer weiteren Distension des Gelenkes, wodurch die intraartikulären Strukturen und vor allem Läsionen des Labrums und des Knorpels noch besser erkennbar werden.

Nach einer intraartikulären KM-Gabe von etwa $10-20 \mathrm{ml}$ einer $2 \mathrm{mmol}$ Gadoliniumlösung werden im Rahmen der MRArthrographie T1-gewichtete Spinechosequenzen und/oder 3-D-T1-gewichtete Gradientenechosequenzen verwendet [3-5, 7, 8, 12, 16-18]. Um Fehlinterpretationen - hervorgerufen durch Fettgewebe - zu vermeiden, sind Sequenzen mit Fettunterdrückung nach intraartikulärer KM-Applikation sehrvorteilhaft [6]. Diese Sequenzen werden in koronaler, axialer oder in schräg-koronaler und schrägsagittaler Ebene angefertigt [3,4]. Die

Radiologe 2019·59:234-241 https://doi.org/10.1007/s00117-018-0471-8

(c) Der/die Autor(en) 2018

\section{Czerny $\cdot$ C. Chiari $\cdot$ I. Nöbauer-Huhmann}

\section{Labrumpathologie des Hüftgelenkes}

\section{Zusammenfassung}

Die Magnetresonanztomographie (MRT) des Hüftgelenkes kommt zur Diagnostik bei vielen klinischen Zustandsbildern zum Einsatz. Zur Abklärung von Pathologien des Hüftgelenkes wird einerseits die konventionelle MRT und anderseits die MR-Arthrographie verwendet. Knochenmarkläsionen, die Osteonekrose, differenzialdiagnostisch die aktivierte Arthrose sowie entzündliche und tumoröse Veränderungen werden mit der MRT zum Teil auch nach intravenöser Kontrastmittelgabe untersucht. Die MR-Arthrographie, bei welcher es durch die intraartikuläre Applikation verdünnten Kontrastmittels zu einer Distension des Gelenkes kommt, ist exzellent zur Abklärung von Läsionen des Labrum acetabulare, Knorpelschädigungen und freien Gelenkskörpern geeignet. In neuerer Zeit erfolgt die MR-Arthrographie mit Traktion der zu untersuchenden Extremität, da es hierdurch zu einer deutlichen Distension der Gelenkskonstituenten kommt und hierdurch vor allem auch Läsionen des Labrums mit Knorpelbeteiligungen noch besser erfasst werden können. Die folgende Arbeit soll einen Überblick über die magnetresonanztomographischen Bilder und ihre Genauigkeit zur Erfassung labraler Läsionen und den Einfluss auf das therapeutische Vorgehen geben.

\section{Schlüsselwörter}

Magnetresonanztomographie $\cdot$ Magnetresonanzarthrographie - Labrumläsion . Knorpelschaden · Klassifikation · Hüftgelenk

\section{Labral pathologies of the hip joint}

\section{Abstract}

Magnetic resonance imaging (MRI) of the hip joint is performed for the diagnostics of many clinical pathologies. For clarification of pathologies of the hip joint conventional MRI and MR arthrography are used. The use of MRI either with or without the intravenous administration of contrast material is performed to diagnose bone marrow lesions, necrosis of the femoral head, inflammatory and tumorous lesions affecting the hip joint and its components. After the intra-articular administration of a diluted solution of contrast material, which results in distension of the joint, MR arthrography is performed for the diagnosis of intra-articular pathologies, such as lesions of the labrum acetabulare, chondral lesions and free intra-articular bodies. In recent times, MR arthrography is carried out with continuous traction of the limb to be examined because this leads to a distension of the joint and its components and in this way particularly lesions of the labrum with involvement of cartilage can be visualized even better. This article gives an overview of MRI and the accuracy of detecting lesions of the labrum acetabulare and the influence on the therapeutic approach.

\section{Keywords}

Magnetic resonance tomography · Magnetic resonance arthrography - Acetabular labral lesions · Chondral lesions · Classification · Hip joint
Positionierung der koronalen und axialen Schichten erfolgt parallel der Körperhauptachsen, jene der schräg-koronalen Schichten erfolgt senkrecht auf die Azetabulumöffnung, die der schräg-sagittalen Schichten parallel zum Femurhals (• Abb. 1; [3]).

Zusätzlich können nach intraartikulärer KM-Gabe noch radiale Sequenzen bzw. Rekonstruktionen durchgeführt werden, welche in der sagittalen Ansicht eine Einteilung der Labrumläsion nach dem Uhrzeigersinn erlauben und so für den Operateur für die Arthroskopie eine bessere und leichtere Zuordnung erlauben $[1,6,22]$.

Eine zusätzliche flüssigkeitssensitive fettgesättigte Sequenz dient dazu, Knochenmarkläsionen darzustellen, und zeigt sensitiv umgebende Weichteilveränderungen. $\mathrm{Zu}$ beachten ist, dass in der (robusten und bei größerem „field of view" gerne verwendeten) Inversionrecovery-Sequenz die gadoliniumhaltige Gelenkflüssigkeit hypointens ist. Alternativ kann beispielsweise eine T2- 


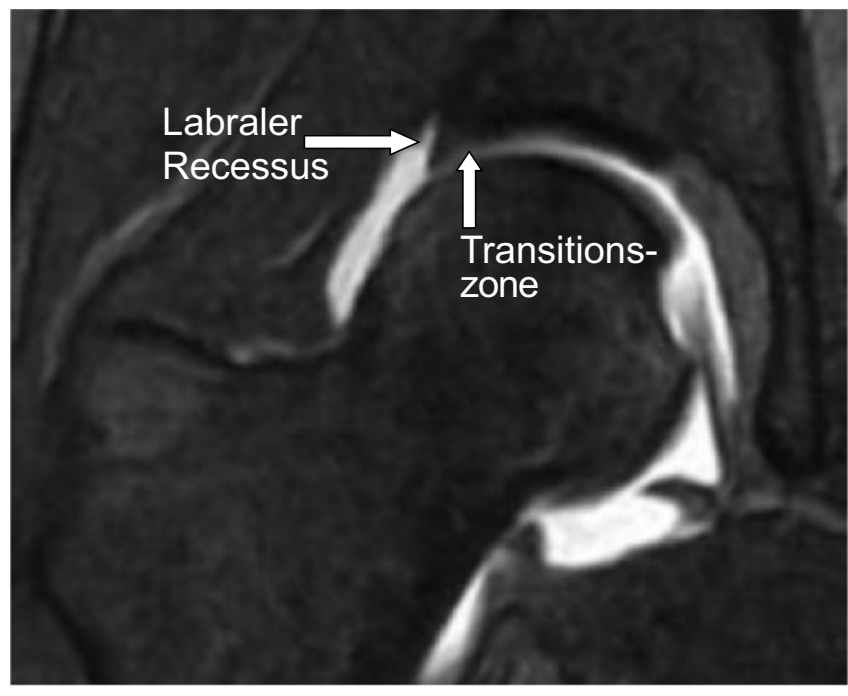

Abb. $3<$ Koronale Magnetresonanzarthrographie eines normalen Labrums

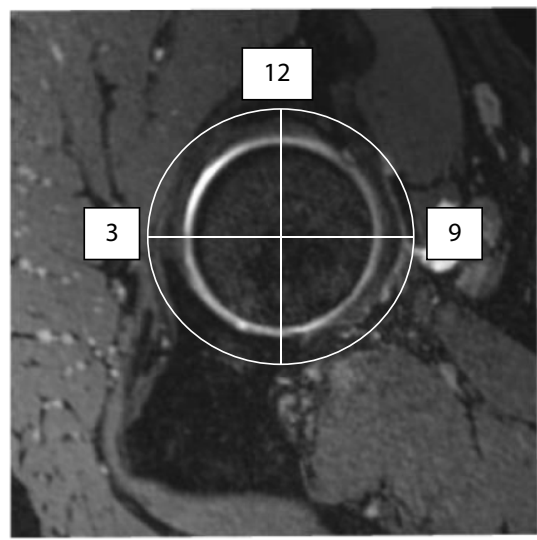

Abb. 4 \& Schemazeichnung zur Lokalisation der Labrumläsion im Uhrzeigersinn (3 Uhr anterior, 12 Uhr oben, 9 Uhr posterior)
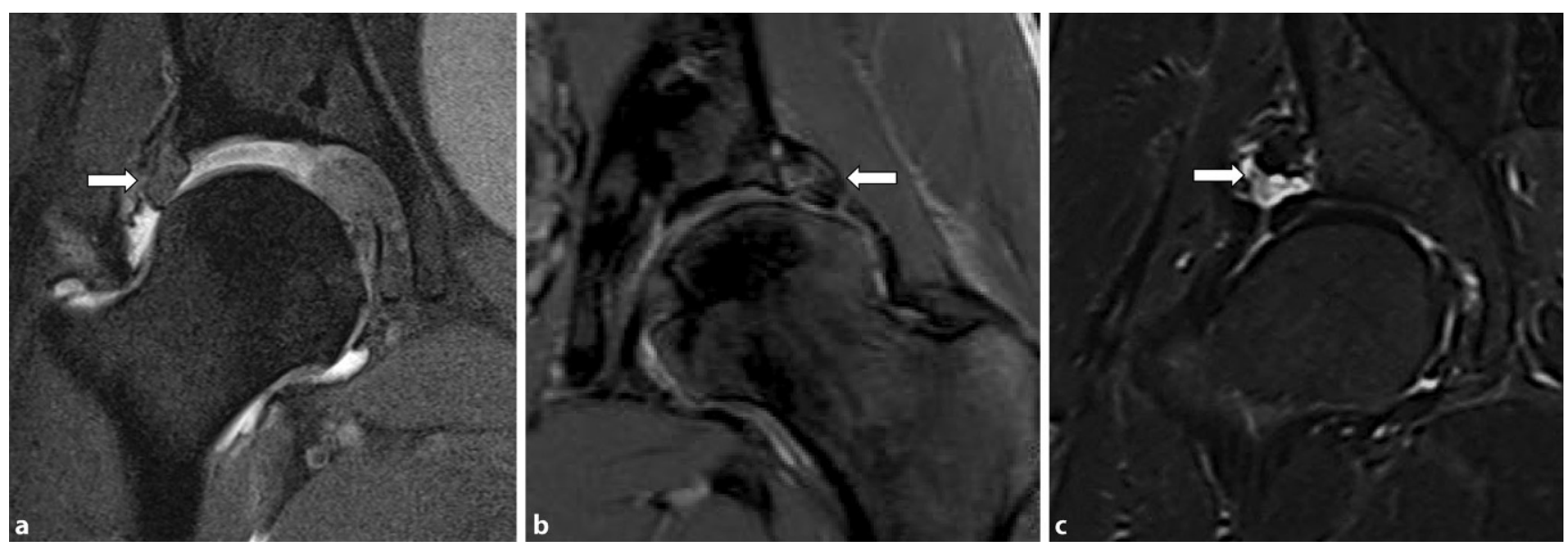

Abb. 5 \& a Koronale MR-Arthrographie mit Traktion eines degenerativ veränderten Labrums (Pfeil).Zusätzlich zeigt sich noch eine synoviale Proliferation. $b$ Das koronale MRT zeigt ein deutlich verplumptes Labrum mit zentralen Signalalterationen entsprechend einer Labrumdegeneration IB (Pfeil). c Koronales MRT eines gering degenerativ veränderten Labrums mit einer paralabralen Zyste (Pfeil)

gewichtete fettgesättigte Sequenz durchgeführt werden.

In der konventionellen MRT werden das Knochenmark und Gelenksveränderungen dargestellt.

Auf den MR-arthrographischen Sequenzen lassen sich Knorpeldefekte durch eine in dem Defekt gelegene KMAnsammlung erfassen [10, 11]. Durch die MR-Arthrographie unter Verwendung der Traktion sind aufgrund der noch besseren Erkennbarkeit von Knorpelschäden die Erfassung dieser noch besser möglich [19, 20].

Sollte eine Kontraindikation zur MRT bestehen, wäre auch die Erfassung von Labrumläsionen durch die CT-Arthrographie möglich. Hierbei werden
$10-20 \mathrm{ml}$ verdünntes jodhältiges $\mathrm{KM}$ direkt in das Gelenk injiziert.

\section{MR-arthrographische Befunde und Klassifikation der Labrumläsionen}

Ein normales Labrum zeigt eine dreieckige Form, manchmal kann es auch etwas flacher oder leicht rundlich imponieren (- Abb. 2 und 3; [4]). Es kann in 4 Abschnitte eingeteilt werden: anterior, anterosuperior, posterosuperior sowie posterior. Nützlich zur besseren anatomischen Zuordnung hat sich die Benennung nach dem Uhrenzifferblatt erwiesen, mit $12 \mathrm{Uhr}$ superior und $3 \mathrm{Uhr}$ anterior sowie 9 Uhr posterior, wobei sowohl bei der rechten wie auch der linken Hüfte die 3 Uhr Position ventral ist ([1, 22]; - Abb. 4).

Das Labrum ist von homogen niedriger Signalintensität und ist direkt am seitlichen Rand des Azetabulums befestigt. Dieses Attachment besteht aus fibrokartilaginärem Gewebe. Zwischen Labrum und Gelenkskapsel befindet sich der Recessus labralis.

Zur Einteilung der Labrumläsionen existieren unterschiedliche Klassifikationssysteme, jenes von Czerny und die modifizierte MAHORN(Multicenter Arthroscopy of the Hip Outcomes Research Network)-Klassifikation nach Schmaranzer ([3, 20]; - Abb. 2). Diese berücksichtigen Form und Größe des Labrums, Alterationen in der Substanz des Labrums, Risse im labralen Attachment 

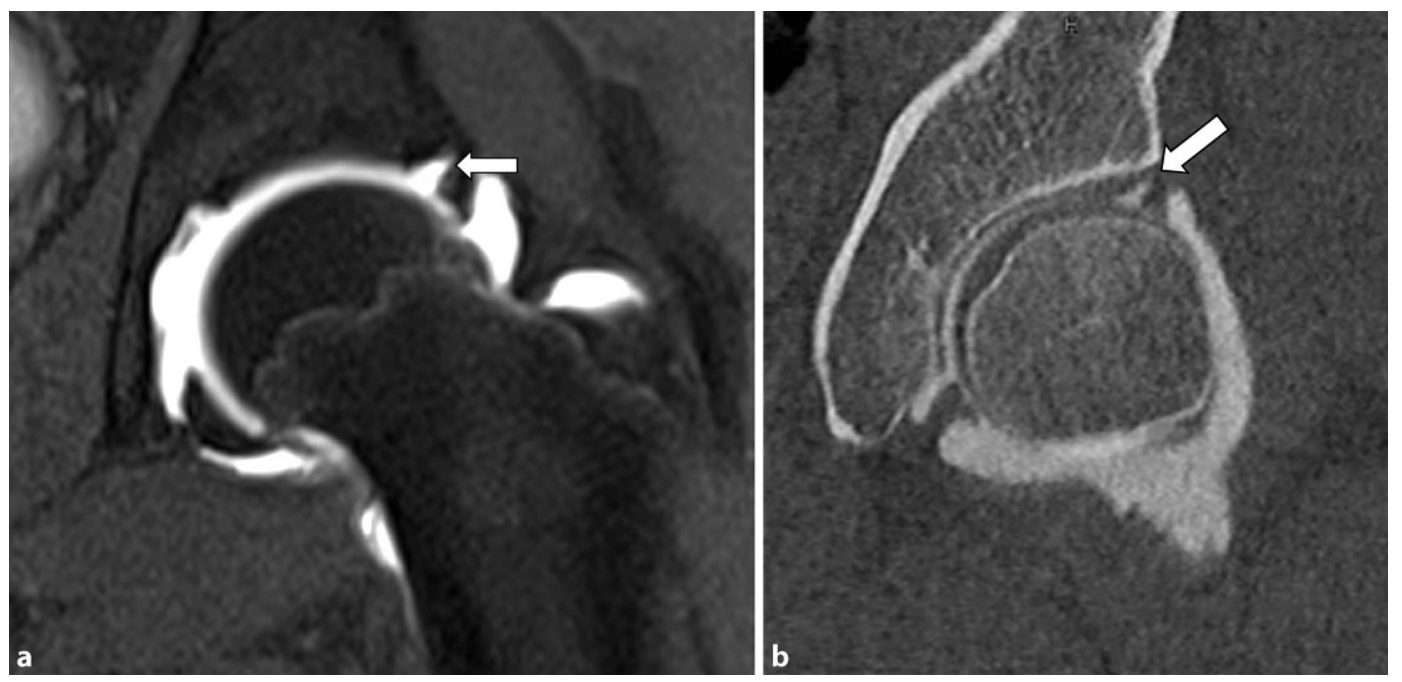

Abb. $6 \triangleleft$ a Koronale MR-Arthrographie eines eingerissenen Labrums (Pfeil). b Koronale Computertomographie-Arthrographie eines Labrumrisses (Pfeil)
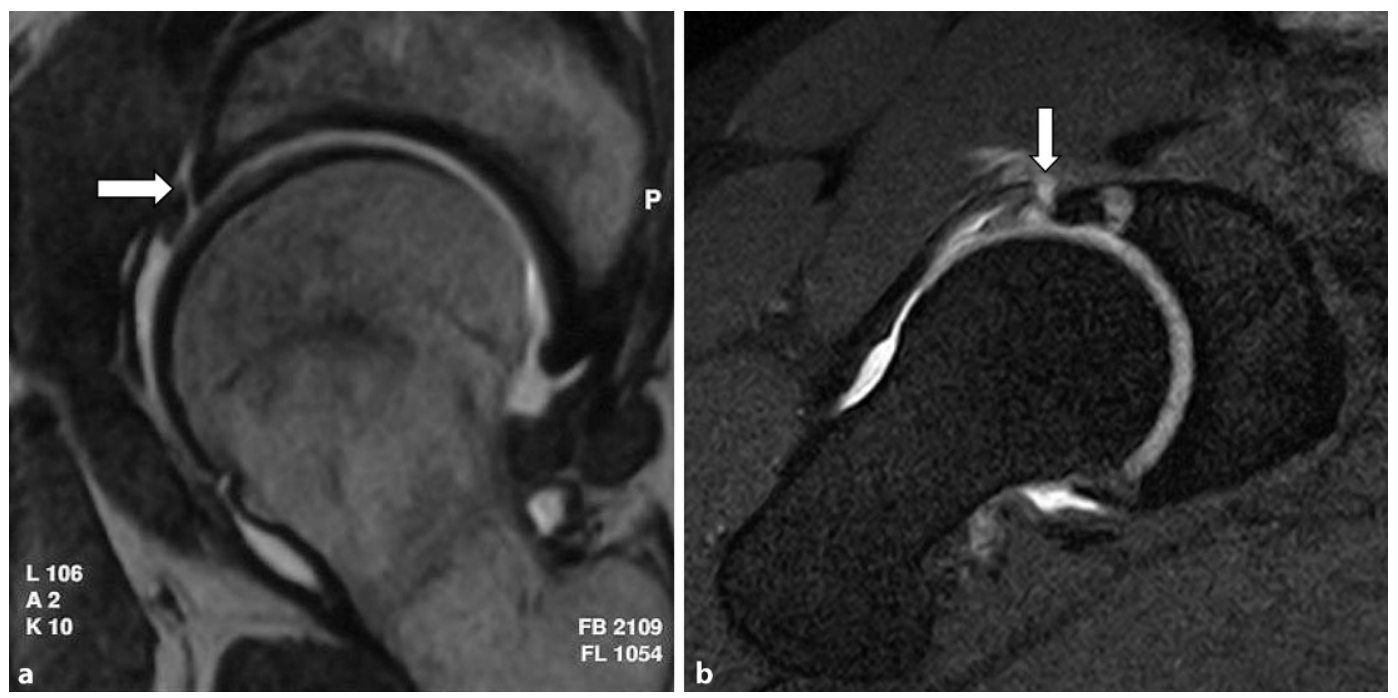

Abb. $7<$ a Schräg-sagittale MR-Arthrographie eines abgerissenen Labrums (Pfeil). b Schräg-sagittale MR-Arthrographie eines Labrumknochenabrisses (Pfeil) mit Kapselruptur

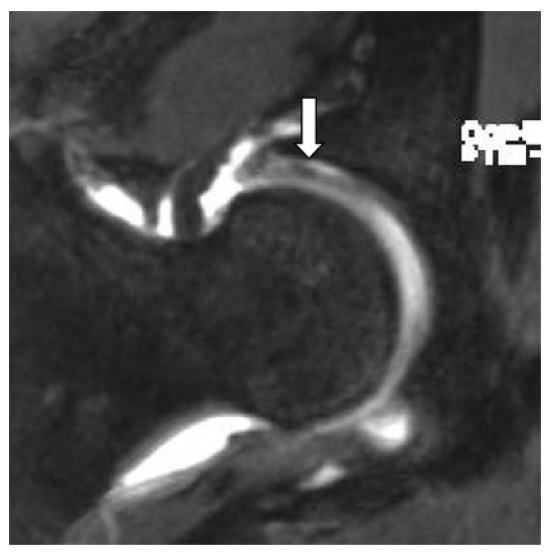

Abb. 8 Radiale MR-Arthrographie mit Traktion eines Labrumrisses in Kombination mit einer Knorpeldelamination (Pfeil) am Azetabulum, assoziierte knöcherne Azetabulumalterationen und Knorpelläsionen sowie Kombinationen dieser Veränderungen. Auf die verschiedenen Knorpelläsionen wird in dieser Arbeit aus Gründen der Übersichtlichkeit nicht eingegangen.

Die erste, auch heute noch sehr gebräuchliche Einteilung nach Czerny unterscheidet daher einerseits Labrumformen und graduiert andererseits die Labrumläsionen (• Abb. 5, 6 und 7): Grundsätzlich werden 2 verschiedene Labrumformen (Typ A und B) voneinander unterschieden. Beim Typ A imponiert das Labrum dreieckig und eher zart, beim Typ B ist es verplumpt, sodass der Rezessus fallweise nicht entsprechend entfaltbar ist.
Sowohl beim Typ A als auch B lassen sich die Labrumläsionen in 3 Grade (I Degeneration, II - Einriss, III - Abriss) einteilen.

Bei der Labrumläsion Typ I (Labrumdegeneration) zeigen sich mukoide Substanzveränderungen entsprechend einer zentralen Signalanhebung (• Abb. 5a). Diese Veränderung lässt sich aber auch bereits in der konventionellen MRT erfassen (• Abb. 5b; [13]). Zusätzlich zu der Degeneration können aber auch noch intralabrale oder paralabrale Zysten vorhanden sein (• Abb. 4c).

Die Labrumläsion Typ II (Labrumeinriss) ist in der MRT durch eine lineare Signalanhebung, welche bis an den Rand des Labrums verfolgbar ist, charakterisiert. Auf MR-arthrographischen Bildern sowie auch bei der CT-Arthrographie 

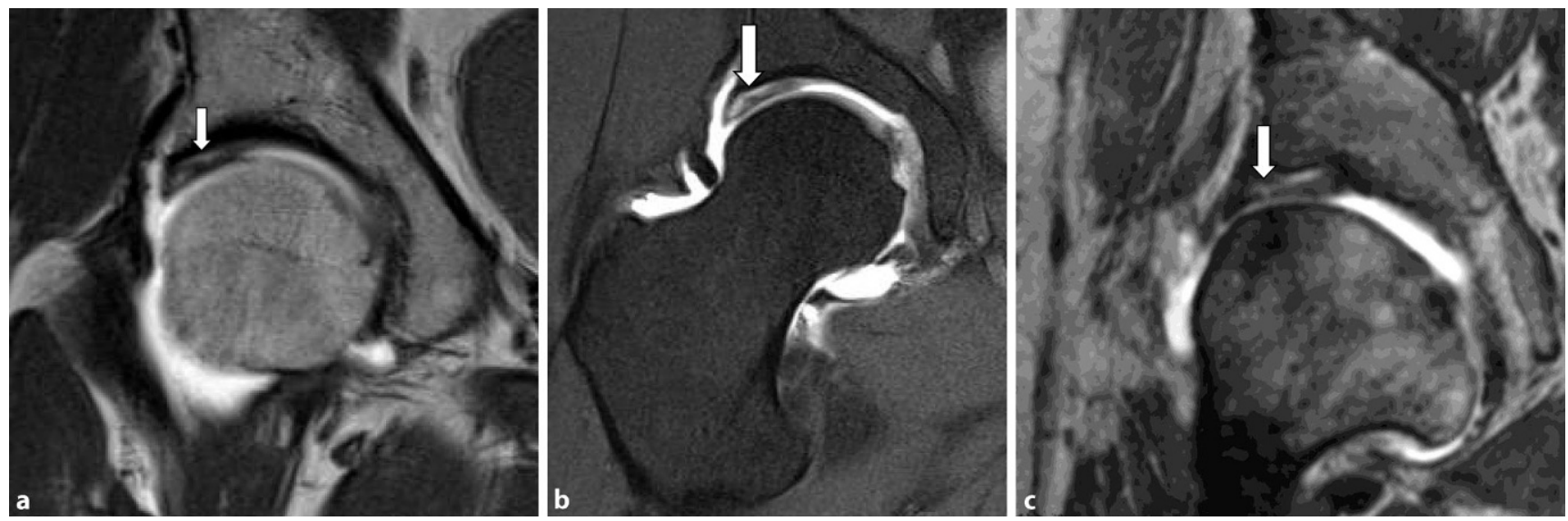

Abb. $9 \Delta$ a Koronale MR-Arthrographie mit Traktion einer Knorpeldelamination (Pfeil). b Koronale MR-Arthrographie mit Traktion und einer Knorpeldelamination mit zusätzlich intralabralem Riss (Pfeil). c Koronale MR-Arthrographie ohne Traktion einer Knorpeldelamination mit komplexer Rissbildung (Pfeil) eines verdickten Labrums bei einer Dysplasiehüfte

lässt sich in diesen Fällen eine KM-Ansammlung, die vom Rand bis in das Labrum verfolgbar ist, erkennen (• Abb. 6). Bei Patienten mit Hüftgelenksarthrose vor allem beim Vorliegen eines Ergusses und hierdurch bedingter Distension und Kontrastierung - können Risse schon in der konventionellen MRT erkennbar sein [9].

Bei der Labrumläsion Typ III (Labrumabriss) findet sich in der MRT eine lineare Signalanhebung, welche das Labrum vom Azetabulum trennt. MRarthrographisch wird das Labrum von KM umspült und ist vom Azetabulum distendiert (๑Abb. 7a). Ein Labrumabriss kann aber in manchen Fällen, welche auch durch schwere Traumata verursacht werden, sogar auch mit einem LabrumKnochen-Abriss und einer Kapselruptur einhergehen ( $\bullet$ Abb. 7b).

Aufgrund der MR-Arthrographie unter Verwendung der Traktion und hierdurch noch besseren Erkennbarkeit von Labrum-Knorpel-Läsionen wurde die oben beschriebene [3-5] - bislang häufig verwendete Klassifikation der Labrumläsionen durch die sog. modifizierte MAHORN-Klassifikation, welche LabrumKnorpel-Läsionen miteinschließt, erweitert $[3,5,20]$. Die modifizierte MAHORN-Klassifikation nach Schmaranzer berücksichtigt - neben den Labrumläsionen - auch Knorpelschädigungen, auf welche in dieser Arbeit aufgrund der Übersichtlichkeit nur vereinzelt - wie z.B. die Delamination - und nicht de- tailliert eingegangen wird. Die detaillierte Übersicht ist in Abb. 2 ersichtlich.

\section{Interpretation und klinische Relevanz der MR-arthro- graphischen Befunde}

Die konventionelle MRT zeigt labrale Veränderungen deutlich schlechter als die MR-Arthrographie [3-5, 8]. Die Degeneration des Labrums (Labrum Typ I nach Czerny) stellt sich sowohl in der MRT als auch in der MR-Arthrographie als intralabrale Signalinhomogenität dar und entspricht pathohistologisch einer mukoiden Verquellung. Der Einriss (Labrum Typ II nach Czerny) stellt sich als Flüssigkeitsmarkierung dar, welche MR-arthrographisch deutlich besser erkennbar ist als mit der konventionellen MRT. Der Einriss ist mit der MR-Arthrographie besser erkennbar als mit der konventionellen MRT, da durch die intraartikuläre KM-Gabe eine Distension des Gelenkes erfolgt und das KM den Einriss markiert [3, 4]. Da es manchmal aber - eventuell aufgrund einer sehr straffen Gelenkskapsel -zu keiner KMMarkierung des Risses kommen kann, kann durch die zusätzliche Traktion der Gelenksspalt erweitert werden und eine KM-Markierung des Risses oder Abrisses möglich sein [20].

Der Labrumeinriss entspricht somit in der Einteilung nach Czerny einer GradII-Läsion. Die modifizierte MAHORNKlassifikation nach Schmaranzer unterteilt diese Rissform weiter in eine chon- drolabrale Separation, einen partiellen Riss vom azetabulären Attachment sowie einen Intrasubstanzeinriss und komplexen Riss.

Das abgerissene Labrum (Labrum Typ III) wird durch Flüssigkeit (Gelenkserguss bzw. intraartikuläres $\mathrm{KM}$ ) vom knöchernen Azetabulum abgegrenzt bzw. umspült. Dies entspricht nach der modifizierten MAHORN-Klassifikation dem kompletten Riss [20].

$\mathrm{Da}$ in den letzten Jahren zunehmend die MR-Arthrographie unter der Verwendung der Traktion der betroffenen Extremität durchgeführt wird, wurde von einigen Autoren wegen der noch besseren Erkennbarkeit von Knorpelschädigungen auch auf diese eingegangen [20]. Die modifizierte MAHORN-Klassifikation nach Schmaranzer unterscheidet hier zwischen einer Knorpelabhebung vom Knochen mit subchondraler „Blase“ und einer Delamination. Durch diese Technik und die Verwendung einer radialen MRArthrographiesequenz/-rekonstruktion lässt sich beispielsweise ein Labrumriss in Kombination mit einer Knorpeldelamination sehr gut erfassen (• Abb. 8 und 9a, b). Aber auch die MR-Arthrographie ohne Traktion ermöglich die Darstellung einer Knorpeldelamination mit einem komplexen Riss ( $\bullet$ Abb. 9c).

$\mathrm{Da}$ in den letzten Jahren zunehmend die arthroskopische Hüftgelenksuntersuchung und -operation zum Einsatz kommt, ist es für den Operateur hilfreich, wenn die Labrum-(Knorpel-)Läsion im Uhrzeigersinn erfolgt $[1,22]$. 
Unter dem Blickwinkel der klinischen Fragestellungen kann somit gesagt werden, dass die MR-Arthrographie des Hüftgelenkes eine trotz ihrer Invasivität relativ einfache und effektive Methode zur Erfassung von Labrumläsionen und freien Gelenkskörpern ist. Die Sensitivität des Nachweises von Labrumläsionen im Vergleich mit den operativen Ergebnissen wird mit $92-95 \%$ bei der MRArthrographie bzw. mit ungefähr $65 \%$ bei der konventionellen MRT angegeben [3-5, 18]. Die MR-Arthrographie rechtfertigt somit ihren etwas höheren Aufwand, die Invasivität und die gering höheren Kosten.

Aufgrund der möglicherweise noch besseren Erkennbarkeit von Labrumläsionen und Knorpelschädigungen, welche auch mit einer Labrumläsion kombiniert sein können, dürfte sich auch der zusätzliche Aufwand der MR-Arthrographie unter Verwendung von Traktion rechtfertigen $[19,20]$.

Da jedoch die MR-Arthrographie mit Traktion bisher noch nicht überall standardmäßig durchgeführt wird, stellt sich die Frage welche Einteilung zu benützen ist.

Auch ohne Traktion lassen sich Knorpel-Labrum-Läsionen - sofern ausreichend intraartikuläre KM-Lösung appliziert wurde - in manchen Fällen diagnostizieren.

Welche Einteilung verwendet werden sollte, wird vermutlich noch in den verschiedenen radiologischen und orthopädischen Gesellschaften diskutiert werden, um dann möglicherweise zu einer konsensuellen Lösung zu kommen.

\section{Fazit für die Praxis}

- Die MR-Arthrographie des Hüftgelenkes sollte aufgrund der Invasivität der intraartikulären Kontrastmittelgabe nur bei Patienten, welche klinisch entsprechend untersucht wurden und bei welchen bereits eine konventionelle MRT des Hüftgelenkes zum Nachweis oder Ausschluss anderer Pathologien als jener des Labrums erfolgte, durchgeführt werden.

- Die intraartikuläre Kontrastmittelgabe ermöglicht durch die Distension der Gelenksstrukturen eine bessere
Darstellung und Abgrenzbarkeit des kapsulär-labralen Komplexes und die Erfassung möglicher Pathologien desselben.

- Die zusätzliche Traktion nach intraartikulärer Kontrastmittelgabe bietet eine weitere Distension und somit eine noch bessere Darstellung und hier vor allem auch gleichzeitig vorliegender Knorpelschädigungen, welche nach den neuesten Erkenntnissen auch für die Therapie eine entscheidende Rolle spielen.

- Inwieweit diese Technik der Traktion routinemäßig zum Einsatz kommen wird, wird sich in den nächsten Jahren wahrscheinlich zeigen.

\section{Korrespondenzadresse}

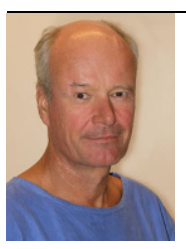

Prof. Dr. C. Czerny Abteilung für Neuroradiologie/Muskuloskelettale Radiologie, Universitätsklinik für Radiologie und Nuklearmedizin, AKH Wien Währinger Gürtel 18-20, 1090 Wien, Österreich christian.czerny@ meduniwien.ac.at

Funding. Open access funding provided by Medical University of Vienna.

\section{Einhaltung ethischer Richtlinien}

Interessenkonflikt. C. Czerny, C. Chiari und I. Nöbauer-Huhmann geben an, dass kein Interessenkonflikt besteht.

Dieser Beitrag beinhaltet keine von den Autoren durchgeführten Studien an Menschen oder Tieren.

Open Access. Dieser Artikel wird unter der Creative Commons Namensnennung 4.0 International Lizenz (http://creativecommons.org/licenses/by/4.0/deed. de) veröffentlicht, welche die Nutzung, Vervielfältigung, Bearbeitung, Verbreitung und Wiedergabe in jeglichem Medium und Format erlaubt, sofern Sie den/die ursprünglichen Autor(en) und die Quelle ordnungsgemäßnennen, einen Link zur Creative Commons Lizenz beifügen und angeben, ob Änderungen vorgenommen wurden.

\section{Literatur}

1. Blankenbaker DG, De Smet AA, Keene JS, Fine JP (2007) Classification and localisation of acetabular labral tears. Skeletal Radiol 36(5):391-397

2. Bsat S, Frei H, Beaule PE (2016) The acetabular labrum. Bone Joint J 98-B:730-735
3. Czerny C, Hofmann S, Neuhold A, Tschauner C, Engel A, Recht MP, Kramer J (1996) Lesions of the acetabular labrum: accuracy of MR imaging and MR arthrography in detection and staging. Radiology 200:225-230

4. Czerny C, Hofmann S, Urban M, Tschauner C, Neuhold A, Pretterklieber M, Recht MP, Kramer (1999) MR arthrography of the adult acetabular capsular-complex: correlation with surgery and anatomy. Am J Roentgenol 173:345-349

5. Czerny C, Kramer J, Neuhold A, Urban M, Tschauner C, Hofmann S (2001) Magnetresonanztomographie und Magnetresonanzarthrographie des Labrum acetabulare: Vergleich mit operativen Ergebnissen. Rofo Fortschr Rontgenstr 173:702-707

6. ESSR (2018)EmpfehlungenderESSR.https://www. essr.org Zugegriffen: 13.11.2018

7. Foti G, Campacci A, Conati M, Trentadue M, Zorzi C, Carbognin G (2017) MR arthrography of the hip: evaluation of isotropic 3D intermediate-weighted FSE and hybrid GRET1-weighted sequences. Radio Med 122(10):774-784

8. Hodler J, Yu JS, Goodwin D, Haghigi P, Trudell D, Resnick D (1995) MR Arthrography of the hip: improved imaging of the acetabular labrum with histologic correlation in cadavers. Am JRoentgenol 165:887-891

9. Jayakar R, Merz A, Plotkin B, Wang D, Seeger L, Hame SL (2016) Magnetic resonance arthrography and the prevalence of acetabular labral tears in patients 50 years of age and older. Skeletal Radiol 45(8):1061-1067

10. Kramer J, Stiglbauer R, Engel A, Prayer L, Imhof H (1992) MR contrast arthrography (MRA) in osteochondrosis dissecans. J Comput Assist Tomogr 16:254-260

11. Kramer J, Recht MP, Imhof H, Stiglbauer R, Engel A (1994) MR arthrography in assessment of cartilage lesions. J Comput Assist Tomogr 18:218-224

12. Leunig M, Werlen $S$, Ungerböck A, Ito K, Ganz R (1997) Evaluation of the acetabular labrum by MR arthrography. J Bone Joint Surg 79:230-234

13. MageeT(2015) Comparison of 3.0-TMRvs 3.0-TMR arthrography of the hip for detection of acetabular labral tears and chondral defects in the same patient population. Br JRadiol 88(1053):20140817

14. Matcuk GR Jr, Price SE, Patel DB, White EA, Cen S (2018) Acetabular labral tear description and measures of pincer and cam-type femoroacetabular impingement and interobserver variability on $3 \mathrm{~T}$ MRarthrograms. Clin Imaging 50:194-200

15. Neuhold A, Czerny C, Wicke I, Liederer M (2002) Detektion freier Gelenkskörper mit der MRArthrographie. AbstractESSR, Valencia

16. Petersilge CA (1997) Current concepts of MR arthrography of the hip. Semin Ultrasound CT MR 18:291-301

17. Petersilge CA (2001) MR arthrography for evaluation of the acetabular labrum. Skeletal Radiol 30(8):423-430

18. Petersilge $C A$, Haque MA, Petersilge WJ, Lewin JS, Lieberman JM, Buly R (1996) Acetabular labral tears: evaluation with MR arthrography. Radiology 200:231-235

19. Schmaranzer F, Klauser A, Kogler M, Henninger B, Forstner T, Reichkendler M, Schmaranzer E (2014) Improving visualization of the central compartment of the hip with direct MR arthrography under axial leg traction: a feasibility study. Acad Radiol 21(10):1240-1247

20. Schmaranzer F, Klauser A, Kogler M, Henninger B, Forstner T, Reichkendler M, Schmaranzer E (2015) Diagnostic performance of direct traction MR arthrography of the hip: detection of chondral and 
labral lesions with arthroscopic comparison. Eur Radiol 25(6):1721-1730

21. Takazawa M, Nakamura J, Abe I, OhtoriS, Watanabe A, Sato $Y$, Sasho T, Orita S, Suzuki M, Motoori K, Masuda Y, Takahashi K, Kishida S (2014) Predictive factors for acetabular labral lesions in osteoarthritis of the hip with radial magnetic resonance imaging - a cross-sectional study. Mod Rheumatol 24(6):974-979

22. Yoon LS, Palmer WE, Kassarjian A (2007) Evaluation of radial-sequence imaging in detecting acetabular labral tears at MR arthrography. Skelet Radiol 36(11):1029-1033

\section{Welche Bakterien sich auf Stethoskopen tummeln}

Stethoskope, die Ärzte in Kliniken mit sich umhertragen, sind offenbar von einer Vielzahl von Bakterien besiedelt. In einer US-Studie fanden sich darunter auch Erreger relevanter nosokomialer Infektionen.

Zur bakteriellen Besiedelung von Stethoskopen existierten bislang nur Studien auf der Basis von Kulturen. Diese sind dadurch begrenzt, dass sie sich auf ganz bestimmte Erreger fokussieren. Um das ganze Spektrum von Keimen zu ermitteln, welche die Membranen von Stethoskopen bevölkern, und zwar speziell von solchen, die auf einer Intensivstation zum Einsatz kommen, hat ein Team der University of Pennsylvania nun einen anderen Ansatz gewählt: das Next-GenerationSequencing.

\section{Next-Generation-Sequencing}

Mit dieser DNA-basierten Methode wurden in zahlreichen Testreihen Abstriche von insgesamt 40 verschiedenen Stethoskopen untersucht: Dazu zählten zum einen Stethoskope die Klinikärzte, Pfleger oder Krankenschwestern um den Hals oder in der Kitteltasche mit sich trugen, zum anderen solche, die im Zimmer des Patienten blieben. Als Vergleich dienten einerseits unbenutzte, also frisch aus der Verpackung entnommene Einmal-Stethoskope, andererseits Blindproben, bestehend aus unbenutzten Tupfern.

\section{Viele unterschiedliche Erreger}

Wie die Autoren Vincent R. Knecht und Kollegen berichten, waren vor allem die vom Klinikpersonal umhergetragenen Stethoskope von einer Vielzahl unterschiedlichster Erreger besiedelt, die von der Haut oder aus dem Verdauungstrakt stammten; darunter, so die Experten, auch Keime, die gemeinhin für nosokomiale Infektionen verantwortlich gemacht werden. Auf jedem der untersuchten Arzt-Stethoskope fanden die Wissenschaftler zum Beispiel Staphylococcus-Spezies. So wurde beispielsweise $S$. aureus auf 24 von 40 Geräten identifiziert. Deutlich mehr als die Hälfte trugen Pseudomonas und Acinetobacter und annähernd $50 \%$ Enterococcus, Stenotrophomonas und Clostridium. Die Arzt-Stethoskope beherbergten deutlich mehr Keime als die Geräte, die im Raum des Patienten blieben, und diese wiederum deutlich mehr als unbenutzte Geräte.

\section{Reinigung nur mäßig wirksam}

Knecht und seine Kollegen untersuchten außerdem den Effekt einer Reinigung: Von 10 Arzt-Stethoskopen, die nach dem empfohlenen Standard desinfiziert worden waren (60 Sekunden mit einem wasserstoffperoxidhaltigen Reinigungstuch abreiben, anschließend trocknen lassen), ging die bakterielle Besiedelung nur in der Hälfte der Fälle auf das Niveau der unbenutzten Stethoskope zurück. Hatte der Arzt sein Stethoskop dagegen nach eigenem Gutdünken gereinigt - etwa mit Wasserstoffperoxid, Alkohol oder Bleichlauge - betrug dieser Anteil nur 10\%.

Welche bakterielle Konzentration auf der Oberfläche eines Stethoskops allerdings für eine potenzielle Übertragung relevanter Keime erforderlich ist, bleibt offen, dieser Zusammenhang wurde in der vorliegenden Studie nicht untersucht. Nicht in der Analyse erfasst wurde zudem mögliches DNAMaterial von Viren oder Pilzen.

Quelle: Ärzte Zeitung basierend auf: (Infect Control Hosp Epidemiol (2018) https://doi.org/10.1017/ice.2018.319 